\title{
MIOCARDIOPATÍA HIPERTRÓFICA OBSTRUCTIVA: MANEJO ANESTÉSICO EN EL PARTO. ROL DE LA ECOGRAFÍA TRANSESOFÁGICA INTRAOPERATORIA
}

\author{
Juan Carlos Devoto G. ${ }^{1}$ y José Luis Troncoso J. ${ }^{2}$
}

Key words: Cardiomyopathy Hypertrophic obstructive, Caesarean Section, General Anesthesia.

\section{INTRODUCCIÓN}

El embarazo en la mujer se distingue por cambios graduales y continuos en la fisiología materna que la preparan para el momento del parto. El parto en cambio, se caracteriza por rápidas variaciones hemodinámicas que frente a la coincidencia de patología cardiovascular y anestesia, pueden superar los mecanismos adaptativos maternos.

La Miocardiopatía Hipertrófica Obstructiva es una entidad clínica que hace difícil el manejo anestesiológico de la embarazada durante este período, especialmente cuando se desarrolla la obstrucción dinámica del tracto salida del ventrículo izquierdo, provocada por el movimiento sistólico del velo anterior de la válvula mitral. La utilización cada vez más frecuente de la ecocardiografía transesofágica intraoperatoria en cirugía no cardiaca, que entrega imágenes anatómicas y funcionales en tiempo real, ha permitido que la monitorización de esta patología facilite su diagnóstico y tratamiento.

Se presenta el siguiente caso clínico en el que una paciente con una miocardiopatía hipertrófica se sometió a una cesárea con anestesia general y donde el manejo hemodinámico fue asistido con las imágenes obtenidas con la ecocardiografía transesofágica intraoperatoria.

\section{CASO CLÍNICO}

Paciente mujer de 31 años, propuesta para cesárea electiva a las 37 y media semanas por el diag- nóstico de doble cesárea anterior. Entre sus antecedentes destacaba ser portadora de una miocardiopatía hipertrófica $(\mathrm{MCH})$ obstructiva, diagnosticada en la adolescencia, en tratamiento con atenolol, que fue suspendido al inicio de su embarazo por su cardiólogo. Tenía historia de síncopes que se habían hecho más frecuentes durante el embarazo. Su capacidad funcional que era normal (CF I) al inicio de éste, disminuyó progresivamente hasta presentar al tercer trimestre disnea a pequeños esfuerzos $(\mathrm{CF}$ III).

En la evaluación preoperatoria predominaba en el examen cardiovascular un soplo holosistólico IV/ VI que se irradiaba hacia la axila, sin la presencia de un tercer ruido ni ingurgitación yugular. Su índice de masa corporal era de $31 \mathrm{~kg} \mathrm{~m}^{-2}$. Sus exámenes de laboratorio eran normales, destacando una hemoglobina de $12 \mathrm{~g} \mathrm{dL}^{-1}$, un Holter ECG normal y un ecocardiograma efectuado a las 30 semanas que mostraba un ventrículo izquierdo hipertrófico con un gradiente transaórtico de $19 \mathrm{~mm} \mathrm{Hg}$ (valor normal: $0-4 \mathrm{mmHg}$ ), una fracción de eyección de $65 \%$ (valor normal: $>55 \%$ ), un doppler transmitral con una onda E: $0,66 \mathrm{~m} / \mathrm{s}$ y una onda A: $0,93 \mathrm{~m} / \mathrm{s}$ con una relación E/A: 0,71 (valor normal: 1-2) y un grosor del septum interventricular de $31 \mathrm{~mm}$ de espesor (valor normal: 6-11 mm), además de una insuficiencia mitral leve con presencia de movimiento sistólico anterior de la válvula mitral (systolic anterior motion, SAM por sus sigla en inglés). Se premedica con famotidina $20 \mathrm{mg}$ dos horas previas a la cirugía.

$\mathrm{Al}$ ingreso al pabellón quirúrgico se monitoriza

Departamento de Anestesiología. Clínica Las Condes. Santiago de Chile.

2 Departamento de Obstetricia y Ginecología. Clínica Las Condes. Santiago de Chile. 
con oximetría de pulso, presión arterial invasiva y electrocardiografía continua de tres derivaciones, que mostraban una frecuencia cardiaca normal. Se colocó un catéter epidural lumbar G18 en el espacio $\mathrm{L}_{2}-\mathrm{L}_{3}$ para proporcionar analgesia postoperatoria y se induce anestesia general endovenosa en modo TCI (target-controlled infusión) con propofol y remifentanil, para una concentración sitio efecto de 2,6 ug ml-1 y $4,5 \mathrm{ng} \mathrm{ml}^{-1}$, respectivamente, como relajante muscular se utilizó rocuronio $0,6 \mathrm{mg} \mathrm{kg}^{-1}$. Inmediatamente de realizada la intubación endotraqueal se instaló un transductor multiplanar (3-8 Mhz) de ecocardiografía transesofágica (FujiFilm Sonosite Edge $\left.{ }^{\circledR}\right)$ para monitorización intraoperatoria, obteniéndose mediciones basales de fracción de eyección y de precarga, la primera en un plano medio esofágico, visión de cuatro cámaras con método de Simpson, constatándose una fracción de eyección de 55\% y la segunda en una visión transgástrica profunda en un eje corto midiendo el área de fin de diástole del ventrículo izquierdo como indicador del volumen de fin de diástole ${ }^{1}$, siendo este de $9 \mathrm{~cm}^{2}$ (valor normal: 8-14 $\mathrm{cm}^{2}$ ) Durante la cirugía se mantuvo una vigilancia continua del tracto de salida del ventrículo izquierdo (TSVI) a través de la visión de cuatro cámaras a $0^{\circ}$ y $130^{\circ}$ para la detección precoz de SAM (Figura 1). El desarrollo de la cirugía transcurrió sin incidentes hasta el momento del alumbramiento. Luego de la administración de oxitocina endovenosa (syntocinon $\left.{ }^{\circledR}\right) 2$ UI, presentó hipotensión sistólica de $65 \mathrm{~mm} \mathrm{Hg}$ con una frecuencia cardiaca de 125 latidos por minuto. Las imágenes ecocardiográficas en el eje corto intragástrico mostraban una disminución del área de fin de diástole del ventrículo izquierdo de menos de $8 \mathrm{~cm}^{2}$ y en el plano medio esofágico visión

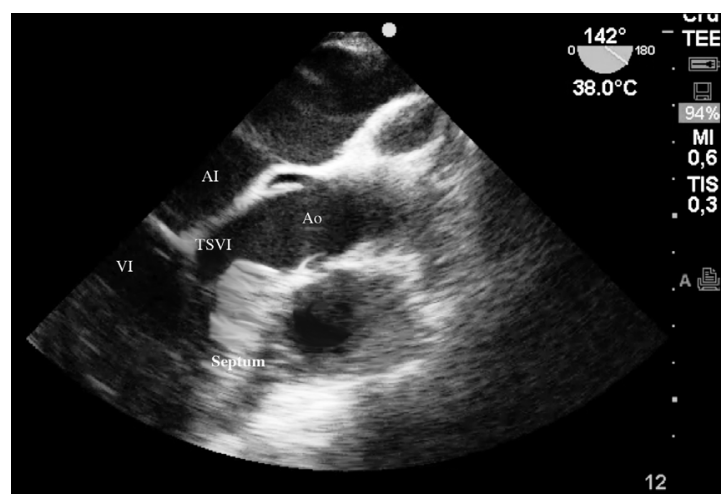

Figura 1. Imagen ecocardiográfica en esófago medio a $142^{\circ}$ que muestra la hipertrofia septal y tracto salida ventrículo izquierdo (TSVI). Aurícula Izquierda (AI). Ventrículo Izquierdo (VI). Aorta (Ao). de cuatro cámaras una disminución cualitativa del diámetro del TSVI en base al desplazamiento del velo anterior de la válvula mitral sin obstrucción total (Figura 2). El doppler color de la válvula mitral mostraba un jet sistólico de regurgitación que abarcaba dos tercios de la aurícula izquierda. En base a estas imágenes se determinó administrar bolos de cristaloides de $100 \mathrm{ml}$ para mantener un área de fin de diástole ventricular mayor de $10 \mathrm{~cm}^{2}$. Como la frecuencia cardiaca permaneció elevada se inició bloqueo beta mimético con propanolol, en bolos de $0,5 \mathrm{mg}$ hasta un total de $1,5 \mathrm{mg}$ endovenoso, obteniéndose con estas medidas estabilidad hemodinámica. En las imágenes ecocardiográficas se observó nuevamente un normal desplazamiento del velo anterior de la válvula mitral.

Durante el resto de la cirugía la paciente se mantuvo estable, administrándose un volumen total de cristaloides de $18 \mathrm{ml} \mathrm{kg}^{-1} \mathrm{~h}^{-1}$. El sangrado intraoperatorio estimado fue de $350 \mathrm{ml}$.

Es extubada en pabellón a los 65 minutos de iniciada la cirugía, manteniéndose con frecuencia cardíaca y presiones arteriales normales. Es trasladada a un intermedio donde su postoperatorio evoluciona sin complicaciones. Se decide entonces postergar la terapia con atenolol con el fin de no interferir con la lactancia. Es dada de alta al cuarto día postoperatorio.

\section{DISCUSIÓN}

La MCH es una enfermedad genética compleja del músculo cardiaco que habitualmente se manifiesta antes de los 30 años y por esto, con cierta frecuencia, podemos verla en el embarazo; no obs-

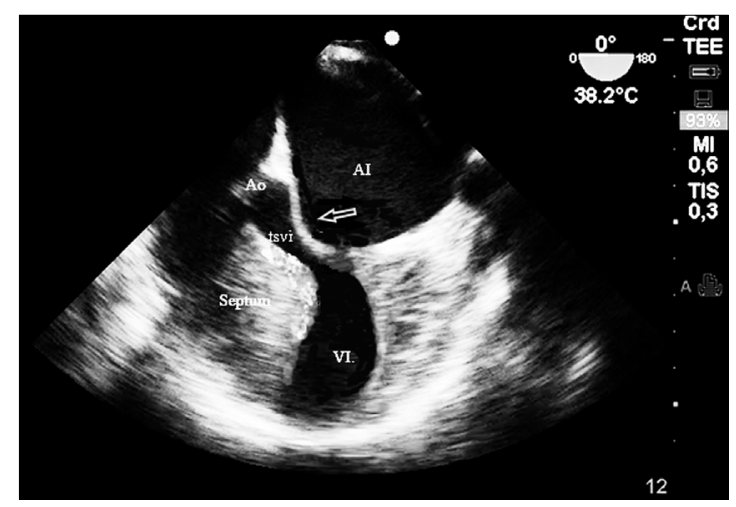

Figura 2. Imagen ecocardiográfica 4 Cámaras $0^{\circ}$, que muestra la obstrucción dinámica del tracto de salida ventrículo izquierdo (TSVI) en sístole. La fecha muestra el velo anterior de la válvula mitral. 
tante ser de curso clínico heterogéneo sigue siendo la causa más frecuente de muerte súbita en jóvenes y atletas. Aunque relativamente común $(0,2 \%)$, a menudo es subdiagnosticada. Se define morfológicamente por una hipertrofia no dilatada del ventrículo izquierdo de causa inexplicada, en ausencia de otra enfermedad sistémica o cardiaca que por sí misma sea capaz de producir la hipertrofia ${ }^{2}$, como por ejemplo, hipertensión arterial o estenosis aórtica.

Ecográficamente se reconoce cuando el espesor de la pared ventricular es mayor o igual a $15 \mathrm{~mm}$, especialmente en presencia de otros factores como historia familiar de MCH. Este engrosamiento de la pared habitualmente es asimétrico comprometiendo con mayor frecuencia el septum interventricular. En este caso, la paciente presentaba un engrosamiento basal del septum de $31 \mathrm{~mm}$, lo que junto a un estado hiperdinámico producido por su embarazo aumentaba la probabilidad de desarrollar una obstrucción del TSVI.

La mayoría de las MCH tienen propensión a desarrollar obstrucción dinámica del TSVI bajo condiciones de reposo o provocadas fisiológicamente. Esto se explica por el desplazamiento del velo anterior de la válvula mitral durante la sístole al ponerse en contacto con el septum interventricular ${ }^{3}$. Esta obstrucción dinámica puede hacerse más evidente en situaciones que disminuyan la precarga e incrementen la contractilidad.

$\mathrm{La}$ anestesia epidural en general no ha sido aconsejada durante el embarazo en mujeres con miocardiopatía hipertrófica debido a su efecto de causar estasis venoso, reducir las presiones de llenado, y potencialmente exacerbar el gradiente ventricular izquierda del tracto de salida ${ }^{4-5}$.

La elección de la mejor técnica anestésica en una paciente portadora de una $\mathrm{MCH}$ es un tema aún no resuelto pero que nos permite un análisis fisiológico más profundo del manejo de esta patología. Existen reportes exitosos de cesáreas efectuadas con anestesia regional con dosis fraccionadas y monitorización de presión venosa central ${ }^{6-7}$. Otros en cambio, recomiendan el empleo de anestesia general ${ }^{8}$. Cualquier técnica que preserve el volumen de fin de diástole y evite el aumento de la contractilidad, ambos factores conocidos en la génesis de SAM, obtendrá buenos resultados. El punto en cuestión es cuál es el óptimo resultado y el menor riesgo. La monitorización intraoperatoria con ecocardiografía transesofágica que nos entrega imágenes de la función cardiaca en tiempo real nos permite una adecuada vigilancia de esos factores, especialmente en lo que se refiere a la precarga.

Cuando analizamos la precarga nos estamos refiriendo al stress de la pared ventricular al final de la diástole (o elongación de la fibra miocárdica) que dependerá del volumen ventricular alcanzado; éste es el parámetro clínico medido con ecografía transesofágica. La forma habitual como se mide hoy la precarga en clínica es extrapolando valores de presiones a volúmenes de llenado ventricular, ya sea a través de la medición de presión venosa central o presión de enclavamiento de capilar pulmonar. Pero la presión y el volumen ventricular tiene una correlación que no es lineal y que depende de la curva de elastancia o distensibilidad miocárdica. Alteraciones de distensibilidad se presentan en pacientes portadores de hipertensión arterial, diabéticos, enfermedad coronaria y muy especialmente en aquellos con alteraciones del grosor de la pared ventricular como es la $\mathrm{MCH}$; en éstos, la monitorización de la presión como parámetro de evaluación de precarga ventricular puede ser de poca utilidad o lo que es peor, llevar a error 9 .

El fenómeno de SAM, referido entre las causas etiológicas de hipotensión intraoperatoria, es poco conocido por anestesiólogos no cardiovasculares y su frecuencia aunque desconocida, se estima que explicaría aproximadamente $3 \%$ de las hipotensiones en las cuales no se logra determinar su causa, lo que también ha sido descrito en pacientes no portadores de $\mathrm{MCH}^{10}$.

El tratamiento está basado en preservar la geometría ventricular ya sea con el aporte de volumen, con el uso de beta bloqueadores o con marcapasos bicamerales ${ }^{2}$.

En el análisis más detallado de este caso clínico, la paciente refería al momento de la evaluación pre anestésica disnea a pequeños esfuerzos (CF III) con un examen ecocardiográfico que mostraba buena función ventricular (contractilidad conservada), con una relación $\mathrm{E} / \mathrm{A}:<1$ característico de una disfunción diastólica grado I y que la gravedad de esta disfunción se vio aumentada con el desarrollo del embarazo. Se estima que alrededor de $40 \%-50 \%$ de las insuficiencias cardiacas pueden tener función sistólica conservada y su fisiopatología está dada por la alteración de la diástole ventricular.

Probablemente si uno analiza la técnica anestésica a utilizar en una insuficiencia mitral debiera inclinarse por las técnicas regionales que reducen la postcarga y mejoran la insuficiencia, pero cuando valoramos en esta paciente los signos ecográfico de severidad, como son el grado de hipertrofia del septum, considerada en este caso como severa, $(>$ $50 \mathrm{~mm}$ es considerada quirúrgica), la presencia de SAM en reposo, la historia de síncopes que aunque de etiología múltiple entre sus causas uno debe considerar también la obstrucción dinámica, sumado a esto los signos de disfunción diastólica, opta- 
mos por realizar la cirugía con una técnica anestésica que no modificara la precarga o la postcarga, que permitiera regular el aporte de volumen en forma más conservadora, evitando sobrecargar a un ventrículo que tolera mal las sobrecargas, más aun considerando que la presencia de disfunción diastólica es un predictor de inestabilidad hemodinámica intraoperatoria y determinante de efectos adversos en cardiocirugía ${ }^{11-12}$.

La administración de oxitocina (2 UI) y la hipotensión secundaria fue el único evento hemodinámico adverso durante la cirugía que causó sólo una obstrucción parcial del TSVI, probablemente debido a la monitorización ecográfica continua. Esto nos debiera plantear si utilizar dosis menores o no utilizar dosis en bolo, especialmente cuando la $\mathrm{ED}_{90}$ de esta droga en cesárea es $0,35 \mathrm{UI}^{13}$.

Finalmente, la monitorización intraoperatoria con ETE si bien no modificó lo que se habría hecho ante una hipotensión secundaria al uso de oxitocina (aporte de volumen), sí permitió evidenciar una precarga adecuada y lo que creemos más importante, permitió objetivar el fenómeno de SAM.

Creemos que su difusión en cirugía no cardiaca aportará con nuevos diagnósticos y mejores tratamientos.

\section{REFERENCIAS}

1. Tousignant C, Walsh F, Mazer D. The use of Transesophageal Echocardiography for preload assessment in critically ill patients. Anesthesia Analgesia 2000; 90: 351-366.

2. Guideline for the diagnosis and treatment of hypertrophic cardiomyopathy. ACCF/AHA. Circulation 2011; 1 24, 2761-2796.

3. Barry JM MD, Chair Jeffrey AT, et al. Contemporary Definitions and Classification of the Cardiomyopathies. Circulation 2006; 113: 1807-1816.

4. Autore C, Brauneis S, Apponi F, et al. Epidural anesthesia for cesarian section in patients with hypertrophic cardiomyopathy: a report of three cases. A nesthesiology 1999; 90: 1205-1207.

5. Minnich ME, Quirk JG, Clark RB. Epidural anaesthesia for vaginal delivery in a patient with idiopathic hypertrophic subaortic stenosis. Anaesthesiology 1987; 67: 590-592.

6. Recansens J, Boada S, Solsona E, et al. Cesárea electiva con anestesia epidural a una gestante con miocardiopatía hipertrófica obstructiva. Rev Esp Anestesiol y Reanim 2000; 47: 320-322.

7. Minich ME, Quirk G, Clark R. Epidural anesthesia for vaginal delivery in a patient with idiophatic hypertrofic subaortic stenosis. Anesthesiology 1987; 67: 590-592.

8. Gomar C, Errando C. Neuroaxial anaesthesia in obstetrical patients with cardiac disease. Current Opinion in Anaesthesiology 2005; 18 : 507-512.

9. Marik P, Baram M, Vahid B. Does Central Venous Pressure Predict Fluid Responsiveness? Chest 2008; 134: 172-178.

10. Cabrera C, Labbe M, Schmied S.
Ecografía transesofágica intraoperatoria y movimiento sistólico anterior de la válvula Mitral: Hallazgo intraoperatorio durante cirugía no cardiaca. Rev Esp Anestesiol Reanim 2007; 54: 440-443.

11. Salem R, Denault A, Couture P. Left ventricular end diastolic pressure is a predictor of mortality in cardiac surgery independently of left ventricular ejection fraction. Br J Anesth 2006; 97: 292-297.

12. Cabrera MC, Schmied S, Vega $\mathrm{R}$, et al. Intraoperative diastolic disfunction as a risk factor of postoperative hemodynamic disfunction. Rev Med Chile 2007; 135: 12761281.

13. Carvalho José CA, Balki, Mrinalini, Kingdom, John, \& Windrim, Rory. (2004). Oxytocin requirements at elective cesarean delivery: a dosefinding study. Obstetrics and gynecology, 104 (5 Pt 1), 1005-1010.

\author{
Correspondencia a: \\ Dr. Juan Carlos Devoto Garcés \\ juandevoto@clc.cl
}

